\title{
TRANSFERENCIA DE TECNOLOGÍA Y CONOCIMIENTO EN EL SECTOR EXPORTADOR DE BOVINOS
}

\author{
Herwy Paternina Pedroza ${ }^{1}$, José Linares Morales $^{2 *}$ y Katerine Hernández Ayala ${ }^{3}$ \\ ${ }^{1}$ Universidad Dr. Rafael Belloso Chacin. Maracaibo, Venezuela \\ ${ }^{2}$ Universidad Politécnica Territorial de Maracaibo. Maracaibo, Venezuela \\ ${ }^{3}$ Universidad del Simú. Cartagena de Indias, Colombia. \\ *Autor de correspondencia: jlinares.iutm@gmail.com
}

Recibido Agosto 2019; Aceptado Noviembre 2019

\begin{abstract}
Resumen - Se formuló una estrategia de transferencia de tecnología y conocimiento en el sector exportador de ganado bovino de Barranquilla, Colombia. La investigación es de tipo descriptiva, con un diseño no experimental, transeccional y de campo; la población y muestra está constituida por 15 profesionales expertos del sector ganadero de Barranquilla agremiados en ASOGANORTE. Se empleó la encuesta como técnica y el cuestionario como instrumento de recolección de datos, constituido por 66 items bajo una escala tipo Likert, con cinco alternativas de respuesta. Se concluye que, la propuesta formulada a través de acciones estratégicas orientadas a la negociación y adaptación tecnológica, como a la asimilación del conocimiento, se convierte en una guía a las empresas del sector exportador de ganado bovino para alcanzar niveles más altos en la curva de aprendizaje organizacional con mayor empoderamiento, consecuencia de la apropiación social del conocimiento tecnológico transferido y capitalizado como parte de su sistema de inteligencia competitiva.
\end{abstract}

Palabras Clave: Estrategia tecnológica; transferencia de tecnologia y conocimiento; adquisición de tecnologia; empresas exportadoras; ganado bovino.

\section{TRANSFER OF TECHNOLOGY AND KNOWLEDGE IN THE BOVINE EXPORT SECTOR}

\begin{abstract}
A technology and knowledge transfer strategy was formulated in the cattle export sector of Barranquilla, Colombia. The research is descriptive, with a non-experimental, transectional and field design; The population and sample is made up of 15 professional experts from the Barranquilla livestock sector who are members of ASOGANORTE. The survey was used as a technique and the questionnaire as a data collection instrument, consisting of 66 items under a Likert-type scale, with five response alternatives. It is concluded that the proposal formulated through strategic actions aimed at negotiation and technological adaptation, as well as the assimilation of knowledge, becomes a guide for companies in the exporting sector of cattle to reach higher levels in the curve of organizational learning with greater empowerment, consequence of the social appropriation of the technological knowledge transferred and capitalized as part of its competitive intelligence system.
\end{abstract}

Keywords: Technological strategy; technology and knowledge transfer; technology acquisition; exporting companies; cattle. 


\section{Transferencia de tecnología y conocimiento en el sector exportador de bovinos}

\section{Introducción}

Desde hace varios años, se viene suscitando en Colombia la oportunidad de exportar ganado a otros países esporádicamente, desde el primer trimestre del 2015, entre los meses de enero y mayo, se han exportado 12.849 animales en tres motonaves; sin embargo, la continuidad de este proceso de proveer animales en pie, puede verse afectada en el corto plazo, teniendo como referente que los proveedores (los ganaderos), no manejan programas de producción constante de animales (Galindo, 2014). Esto se demuestra en los informes de supervisión realizado a las fincas o hatos, donde se evidencia el mal estado de las tierras, el pésimo estado reproductivo de sus animales, el alto intervalo entre partos (tiempo entre un parto y otro), además del inadecuado manejo higiénico-sanitario de los hatos y las heridas que presentan algunos animales (Oviedo et al., 2016).

Las Buenas Prácticas Ganaderas - BPG son normas que se aplican durante el proceso de producción pecuaria, con el fin que la empresa ganadera sea sostenible ambiental, económica y socialmente y de esta manera obtener productos sanos, seguros y de buena calidad. Las BPG son aplicables a todo lo largo de la cadena productiva bovina: desde el productor o eslabón primario, seguido por el transformador hasta que llegue al consumidor final (Espinal et al., 2006). Las normas creadas para el establecimiento de las BPG, pretenden minimizar el impacto que las prácticas pecuarias tienen sobre el medio ambiente, disminuir los riesgos de contaminación de los productos pecuarios con agentes químicos, físicos y biológicos y mejorar tanto el bienestar laboral de los trabajadores rurales, como el bienestar de las especies animales que son explotadas técnicamente (Lombana et al., 2013).

A nivel mundial ha crecido la expectativa por la implementación de BPG, que permita asegurar la continuidad, calidad, sanidad de los animales para sacrificio y posterior consumo humano, teniendo como referente, que un mal manejo en la producción animal, se refleja en la calidad y en la probabilidad de presentarse enfermedades trasmitidas por alimentos-ETA (Díaz et al., 2005), Según el Instituto Nacional de Salud de Colombia (2015) las enfermedades transmitidas por alimentos son un problema de salud pública generalizado y creciente, tanto en los países desarrollados y en desarrollo, desde 2005 han muerto 20 millones de personas por ETAS, razón por la cual cada vez los consumidores son más exigentes en cuanto a la higiene y sanidad de los alimentos (Reyes et al., 2018).

En el caso de la costa Caribe colombiana, existe una organización que agrupa a los productores de la zona, en la búsqueda de mejores condiciones productivas en el marco de las BPG, correspondiente a la Asociación de Ganaderos de la Costa Norte (ASOGANORTE), la cual es una entidad sin ánimo de lucro que representa y defiende los intereses de los ganaderos y las labores que contribuyan al desarrollo del sector rural regional y nacional. Está al servicio de la región apoyando al sector ganadero desde hace 29 años, tiene su estructura organizacional definida con principios de calidad buscando siempre el mejoramiento continuo en todos sus procesos.

La política de empresa de ASOGANORTE, según su página oficial, se basa en la calidad del servicio de existencia técnica integral a sus clientes, ofreciendo un servicio personalizado y ajustado a las diferentes necesidades, trabajando con ellos para formar un equipo enfocado al mismo objetivo. ASOGANORTE está formado por un grupo de profesionales especialistas en sus respectivos sectores, para poder prestar a los clientes la ayuda necesaria a sus proyectos, aportando soluciones útiles y teniendo presente los principios corporativos. 
Ahora bien, ante las eventualidades presentadas en algunas entregas de animales a las empresas exportadoras de ganado, con heridas, mal higiene, y entregas incompletas, es importante establecer líneas de mitigación a eventos adversos, que le permita a estas empresas mejorar la prestación de servicios, la continuidad, calidad y cantidad de los animales. En este sentido, la exportación de ganado macho cebado a países del medio oriente abrió una brecha importante en cuanto a valor por animal en pie en los diferentes municipios del departamento del Atlántico. Una de las principales empresas exportadoras de la región, es la SGS, la cual cuenta con tres (3) personas que coordinan con el comprador la garantía de que el producto embarcado salga del puerto en buen estado, hasta su destino, luego de un viaje de 21 a 26 días de duración. La realidad es que no siempre es posible garantizar la calidad, dado que muchas veces se han embarcado animales con parásitos externos o heridas llenas de gusanos.

Evidentemente, el problema radica principalmente en la inadecuada gestión de la calidad de los productos exportados, dada la no implementación de BPG, en términos de: (1) instalaciones pecuarias y bienestar animal; (2) alimentación y medio ambiente; (3) sanidad animal y bioseguridad; (4) uso de medicamentos veterinarios; (5) saneamiento básico; (6) transporte; (7) registro y documentación; (8) manejo integral de plagas; (9) almacenamiento de insumos pecuarios y agrícolas; (10) trazabilidad; (11) bienestar animal; (12) personal; y (13) transporte (Villarreal, 2015).

Estas prácticas, en la gran mayoría de las unidades productivas bajo ganadería tradicional son desconocidas, o se conocen, pero no se implementan; por lo que la situación problemática pasa a explicarse desde la perspectiva de la tecnología adquirida para llevar a cabo estas BPG y del conocimiento dominado por el sector ganadero que es inadecuadamente transferido. Es así, dada esta situación, como desde SGS o cualquiera de las empresas exportadoras se puede transferir el conocimiento tecnológico a los proveedores que no manejan estas BPG. Entre los síntomas presentes en este contexto, se observa la poca información manejada por los ganaderos en materia de tecnología agropecuaria de apoyo a las BPG. Se evidencia que la gran mayoría de los vendedores de animales no tiene en cuenta programas de mejora genética que permitan hacer los ciclos de vida de las exportaciones de ganado bovino perdurables en el tiempo; al ser productores tradicionales, venden lo poco que tienen sin pensar en la reposición de los animales en el corto y largo plazo.

Por otra parte, en visitas realizadas a campo se nota un total abandono en cuanto a producción de forrajes que permitan garantizar un buen régimen alimenticio, sumado a la baja calidad genética de estos, lo que incide en la baja tasa de reproducción animal, pues si en época de verano no se cuenta con alimento, entonces disminuirá la reproducción de los animales, como de hecho ya está sucediendo. Es aquí donde la transferencia de tecnología juega un papel determinante, pues entre más se tecnifiquen las fincas mejor será el tratamiento del ganado y la productividad, con lo que el proveedor puede precisar cada cuánto tiempo puede estar exportando ganado con excelentes precios, incrementando la rentabilidad del negocio.

En cuanto a los mecanismos de transferencia de tecnología y conocimiento, se observa que no existen instrumentos que permitan determinar la forma en que debe llevarse a cabo el proceso, basado en la identificación de necesidades tecnológicas propias de los proveedores. En este sentido, la primera necesidad es la de transferir genéticamente embriones que permitan la obtención de crías con mayor adaptabilidad a las condiciones ambientales y los requerimientos del proveedor, es decir, si necesita solo animales de color blanco, se trabajará con líneas genéticas que permitan esta característica por ejemplo la 
cebú, que además del color, su rusticidad les permite mayor adaptabilidad a las altas temperaturas de la zona.

De igual manera, se observa la ausencia de programas de cooperación y alianzas tecnológicas con otras empresas que ofrezcan servicios para el desarrollo de la ganadería bovina. Se puede considerar, por ejemplo, en cuanto al tipo de alimentación requerida para el ganado, existen empresas que ofrecen tipos de pastos adaptados a las condiciones ambientales del departamento, tonelaje producido por hectáreas, y tipos de alternativas alimenticias que se puedan realizar con este pasto, como ensilaje, heno, entre otros.

Asimismo, los pocos proveedores que incorporan tecnología, lo hacen sin seguir alguna estrategia o metodología de adquisición, por lo que en ocasiones ciertas expectativas alrededor de la tecnología no son cubiertas, o terminan comprando lo que no necesitan. Estos no cuentan con el asesoramiento oportuno, o la supervisión permanente de actores que faciliten los procesos de adquisición de tecnología y conocimiento. Aun cuando realmente existe un ente territorial como lo es AGROSAVIA, que maneja las certificaciones de empresas que pueden impulsar al sector y llevarlo a la vanguardia de avances tecnológicos en cuanto a mejoras genéticas y de los tipos de pastos que se produzcan en el departamento. La ausencia de cooperación estratégica imposibilita la supervisión y actualización de los mecanismos para la transferencia del conocimiento tecnológico.

Por lo antes expuesto, se hace necesario aprovechar la oferta de animales que la empresa SGS coloca a disposición, y sumado al aporte de los pequeños o medianos productores que se vinculen, se puedan crear pilotos demostrativos para fomentando una cultura de productividad basada en BPG, diferente a la tradicional actualmente presente. Este proceso puede extenderse a toda la región del departamento del Atlántico, dado que Barranquilla no es el único municipio que provee animales; todas sus cabeceras municipales participan en este proyecto, dada la privilegiada posición geográfica dotada de un puerto marítimo con condiciones aptas para el desarrollo del sector exportador de ganado bovino, que promueve un alcance de gran impacto económico para la economía agropecuaria colombiana.

\section{Revisión de la literatura}

En la literatura especializada se encuentran varios planteamientos relacionados con la Transferencia Tecnológica, sin embargo, para los efectos del presente artículo, se fija posición con respecto a la definición del Instituto de Transferencia de Tecnología y Conocimiento, como el movimiento de tecnología y/o conocimiento, desde un proveedor que comercializa la tecnología, hacia un receptor (González-Sabater, 2011; Guerrero \& Urbano, 2012; Wehn \& Montalvo, 2016; Vázquez, 2017).

Otras posturas complementarias la consideran como la transferencia entre diferentes organizaciones del know-how y del capital intelectual, para su uso en el desarrollo de bienes y servicios comercialmente viables, y en el que intervienen además agentes intermediarios que facilitan el proceso (Rubiralta, 2003; Beraza \& Rodríguez, 2010). En el marco de la relación academia - empresa, dentro de los fines mencionados anteriormente, se corresponde al proceso mediante el cual la empresa accede a los avances tecnológicos desarrollados y trasladados desde el ámbito científico (López et al., 2006; Solano et al. 2013).

De manera que, estos procesos de transferencia mejoran la calidad de vida de las personas, gracias a la introducción de innovaciones que optimizan la salud pública, la nutrición y las comunicaciones, así como las políticas que promueven la transferencia de tecnología en materia de educación, y el incentivo al IPSA SCIENTIA - Vol. 4 No 1 - 2019 
desarrollo económico (Reyes, 2010; Finston, 2011). Por medio de la transferencia se propaga el conocimiento, capacidades, elementos técnicos y variedad de equipos (Galindo et al., 2011; Robledo, 2013; Geizzelez \& Linares, 2016; Vargas, 2017) .

Cuando la transferencia se hace desde el exterior, las organizaciones deben tener estrategias que permitan que sus colaboradores generen modelos mentales compartidos, logrando de esta manera un aprendizaje organizacional efectivo (Herrera \& Giraldo, 2010; Avendaño, 2017). De su investigación, León et al. (2009), concluyen que las actividades de transferencia de conocimiento son compatibles con las actividades empresariales (Borbón \& Arvizu, 2015).

Sobre la base de estos planteamientos, la estrategia tecnológica se direcciona al proceso de adquisición tecnológica, siendo que la innovación amerita una demanda de recursos y requieren insumos con los que se crean oportunidades para desarrollar nuevos productos y procesos (Correa, 2012). Por lo tanto, las estrategias tecnológicas hacen explícitas las opciones tecnológicas de la empresa y resultan de una reflexión previa que busca dar respuesta a interrogantes como: ¿en qué estado se encuentra la tecnología?; si está cerca del límite de su vida útil ¿qué alternativas se perciben?; ¿qué nuevas tecnologías pueden impactar la cadena de valor?; ¿qué tecnologías están desarrollando los competidores?; ¿cómo se relaciona la estrategia tecnológica con la estrategia empresarial?, ¿cómo contribuye la tecnología a crear ventajas competitivas en la empresa? (Hidalgo et al., 2002; Linares-Morales \& Geizzelez-Luzardo, 2016). En tal sentido, el objetivo del presente artículo es formular una estrategia de transferencia de tecnología y conocimiento en el sector exportador de ganado bovino de Barranquilla, Colombia.

En conclusión, toda estrategia tecnológica conlleva un conjunto de técnicas sistémicas de pensamiento lateral para generar ideas creativas, que pueden ser aplicadas por una persona para estimular su propio pensamiento creativo o en grupos. Estas técnicas son aplicadas siguiendo un procedimiento dentro de una sesión formal de pensamiento creativo (González 2015; Muñoz, 2018).

\section{Materiales y Métodos}

Atendiendo al grado de conocimiento del objeto de estudio el artículo se considera descriptivo, diseño no experimental, transeccional, de campo (Hernández,et al,, 2014; Hurtado, 2015), ya que a partir del análisis la gestión de tecnología y conocimiento en el contexto de las empresas del sector exportador de ganado bovino en la ciudad de Barranquilla, se procedió con la formulación de una estrategia tecnológica que permita viabilizar dicho proceso.

La población corresponde al conjunto de especialistas de la Asociación de Ganaderos de la Costa Norte (ASOGANORTE) vinculados al sector bovino; así como profesionales con experticia en BPG en empresas exportadoras que operan desde la ciudad de Barranquilla, Departamento del Atlántico. Por su parte, la muestra seleccionada responde a un muestreo no probabilístico intencional (López, 2011).

La inclusión de unidades de análisis para constituir la muestra, obedece a los siguientes criterios: (1) expertos en actividades de exportación de ganado bovino; y (2) actores empresariales que para el momento de realizar la recolección de datos, se encuentren ejecutando tales actividades, o supervisando el cumplimiento de las BPG. Es así como, la muestra se compone de un total de quince (15) sujetos, tal como se observa en el cuadro 1.

IPSA SCIENTIA - Vol. 4 No $1-2019$ 
Cuadro 1: Muestra del estudio

\begin{tabular}{|c|c|c|}
\hline Organismo & Criterio de inclusión considerado & Cantidad \\
\hline ASOGANORTE & Criterios (1) y (2) & 12 \\
\hline SGS & Criterio (3) & 3 \\
\hline \multicolumn{2}{|c|}{ Total de la muestra } & $\mathbf{1 5}$ \\
\hline
\end{tabular}

Fuente: Elaboración propia, basado en datos de ASOGANORTE (2019)

La técnica de recolección de datos utilizada consiste en la observación mediante encuesta; y como instrumento se emplea un cuestionario, conformado por sesenta y seis (66) ítems, bajo una escala tipo Likert construida a partir de cinco (5) alternativas de respuestas, a saber: Siempre; Casi siempre; Algunas veces, Casi nunca y Nunca, valoradas por medio de una puntuación entre cinco y uno, respectivamente. Se utilizó la validez de contenido mediante el juicio de cinco (5) expertos, quienes evaluaron la pertinencia de la variable de estudio sobre los objetivos, dimensión, indicadores y redacción con sus correspondientes ítems; con respecto a la confiabilidad, se realizó una prueba piloto a seis (6) sujetos que no forman parte de la muestra estudiada y que exhiben los mismos criterios de selección de la misma, y fue calculada a través del coeficiente Alfa de Cronbach, cuyo resultado fue de 0.79 , equivalente a una alta confiabilidad.

\section{Propuesta de una estrategia de transferencia de tecnología y conocimiento}

Como consecuencia del análisis e interpretación de los datos obtenidos en el estudio, se procedió a elaborar una propuesta orientada a la formulación de una estrategia de transferencia de tecnología y conocimiento en el sector económico abordado, tal como se planteó en el último objetivo específico de la presente investigación. De esta manera, en el cuadro 2 se muestran los elementos que caracterizan la variable objeto de estudio y a su vez sirven de base para la propuesta en cuestión.

Cuadro 2. Resumen de resultados que caracterizan la propuesta

\begin{tabular}{|c|c|c|}
\hline $\begin{array}{c}\text { Objetivo Específico } \\
\text { (Dimensión) }\end{array}$ & \multicolumn{2}{|c|}{ Elementos base de la propuesta } \\
\cline { 2 - 3 } & Indicadores & Hallazgos \\
\hline $\begin{array}{c}\text { Identificar las modalidades de } \\
\text { transferencia de tecnología y } \\
\text { conocimiento en las empresas } \\
\text { exportadoras de ganado bovino. } \\
\text { (Modalidades de transferencia) }\end{array}$ & $\begin{array}{c}\text { Vía de la transferencia; Formalidad } \\
\text { de la colaboración; Ámbito } \\
\text { geográfico de la colaboración; Tipo } \\
\text { de contraprestación acordada }\end{array}$ & $\begin{array}{c}\text { Modalidad presente: } \\
\text { directa/indirecta; formal/informal; } \\
\text { local/nacional/internacional; } \\
\text { contraprestación económica }\end{array}$ \\
\hline $\begin{array}{c}\text { Determinar los mecanismos de } \\
\text { transferencia de tecnología y } \\
\text { conocimiento en las empresas } \\
\text { exportadoras de ganado bovino. } \\
\text { (Mecanismos de transferencia) }\end{array}$ & $\begin{array}{c}\text { Acuerdos de licencia; } \\
\text { Cooperación tecnológica; } \\
\text { Asistencia técnica y servicios; } \\
\text { empresas; Alianzas tecnológicas; } \\
\text { Adquisiciones y fusiones; } \\
\text { Compra/venta de bienes y equipos }\end{array}$ & $\begin{array}{c}\text { Mecanismo de transferencia } \\
\text { predominante: } \\
\text { Acuerdos de licencia }\end{array}$ \\
\hline $\begin{array}{c}\text { Describir las etapas de adquisición de } \\
\text { tecnologia y conocimiento en las } \\
\text { empresas exportadoras de ganado } \\
\text { bovino. (Etapas de adquisición) }\end{array}$ & $\begin{array}{c}\text { Existencia de necesidad } \\
\text { tecnologica; Elección del } \\
\text { proveedor; Negociación; }\end{array}$ & $\begin{array}{c}\text { Etapas de menos categorización: } \\
\text { Negociación con el proveedor } \\
\text { Implantación de la tecnología }\end{array}$ \\
\hline $\begin{array}{c}\text { Establecer el tipo de estrategia } \\
\text { tecnológica necesaria para las empresas la tecnología } \\
\text { exportadoras de ganado bovino. } \\
\text { (Tipos de estrategia) }\end{array}$ & $\begin{array}{c}\text { Estrategia Ofensiva; Defensiva; } \\
\text { Imitativa; Dependiente; } \\
\text { Tradicional; Oportunista }\end{array}$ & $\begin{array}{c}\text { Estrategia tecnológica } \\
\text { predominante: }\end{array}$ \\
Estrategia Oportunista
\end{tabular}

Fuente: Elaboración propia 
Al revisar la dimensión Modalidades de transferencia se obtuvo que: según la Vía de transferencia y la Formalidad de la colaboración, la misma es directa formal (en el caso de la tecnología) e indirecta informal (en el caso del conocimiento); en cuanto al Ámbito de la colaboración, se extiende en todos los contextos (local, nacional e internacional); y según el Tipo de contraprestación acordada entre las partes, ésta es fundamentalmente económica.

En lo que respecta a la dimensión Mecanismos de transferencia, se tiene que los acuerdos de licencia es el indicador de más alta presencia en las empresas exportadoras consultadas; mientras que para la dimensión Etapas de Adquisición, las de más baja presencia luego del análisis de media aritmética, corresponden a las etapas finales del proceso, es decir, la negociación con el proveedor y la implantación de la tecnología.

Finalmente, considerando la dimensión Tipos de estrategia, se manifiesta como la preponderante la estrategia oportunista, correspondiendo al indicador de más alta valoración en el presente trabajo de grado, dentro de todos aquellos considerados para las diferentes dimensiones que permitieron operacionalizar la variable de investigación.

Sobre la base de estos hallazgos, el investigador procede a plantear unos objetivos estratégicos (ver cuadro 3), así como definir conceptualmente la propuesta como: una estrategia tecnológica oportunista y reactiva, basada en acciones planificadas de negociación y adaptación tecnológica, asi como de asimilación del conocimiento a manera de lineamientos, que persiguen garantizar, de manera efectiva, la transferencia de tecnología y conocimiento, cuyos derechos de propiedad están protegidos bajo acuerdos de licencia para su uso y explotación en las empresas del sector exportador de ganado bovino.

Cuadro 3. Objetivos estratégicos de la propuesta

\begin{tabular}{|c|l|}
\hline DIMENSIÓN & \multicolumn{1}{|c|}{ OBJETIVO GENERAL } \\
\hline Etapas de adquisición & $\begin{array}{l}\text { Garantizar la transferencia de tecnología y conocimiento en las empresas del sector } \\
\text { exportador de ganado bovino mediante el fortalecimiento del proceso de } \\
\text { adquisición de tecnología }\end{array}$ \\
\hline INDICADOR & \multicolumn{1}{c|}{ OBJETIVOS ESPECÍFICOS } \\
\hline $\begin{array}{c}\text { Negociación con el } \\
\text { proveedor }\end{array}$ & $\begin{array}{l}\text { Consolidar las bases de la negociación tecnológica a partir del establecimiento de } \\
\text { acuerdos de licencia como modalidad de adquisición }\end{array}$ \\
\hline $\begin{array}{c}\text { Implantación de la } \\
\text { Tecnología }\end{array}$ & $\begin{array}{l}\text { Incrementar las capacidades tangibles e intangibles del negocio exportador } \\
\text { mediante una efectiva adaptación tecnológica }\end{array}$ \\
\cline { 2 - 2 } & $\begin{array}{l}\text { Fomentar la apropiación social del conocimiento tecnológico dada la asimilación } \\
\text { del mismo como elemento de inteligencia competitiva }\end{array}$ \\
\hline
\end{tabular}

Fuente: Elaboración propia

Objetivo Estratégico No. 1: Consolidar las bases de la negociación tecnológica a partir del establecimiento de acuerdos de licencia como modalidad de adquisición (ver cuadro 4).

Cuadro 4 Acciones de la estrategia de negociación

\begin{tabular}{|c|c|}
\hline PROCESO CLAVE & \multicolumn{1}{|c|}{ ACCIONES ESTRATÉGICAS } \\
\hline & $\begin{array}{c}\text { Realizar procesos de vigilancia basado en necesidades tecnológicas para } \\
\text { seleccionar mecanismos de transferencia idóneos. }\end{array}$ \\
$\begin{array}{c}\text { Negociación de } \\
\text { tecnología }\end{array}$ & $\begin{array}{c}\text { Formalizar contratos tecnológicos de acuerdo con el mecanismo de } \\
\text { transferencia adoptado. }\end{array}$ \\
& $\begin{array}{c}\text { Establecer acuerdos sobre apropiación del conocimiento o confidencialidad, en } \\
\text { los casos de aplicación exclusiva a una empresa del sector. } \\
\text { Actualizar las capacidades cientifico-tecnológicas del negocio gracias al } \\
\text { conocimiento involucrado en los acuerdos de licencia. }\end{array}$ \\
\hline
\end{tabular}

IPSA SCIENTIA - Vol. 4 Nº $1-2019$ 


\section{Transferencia de tecnología y conocimiento en el sector exportador de bovinos}

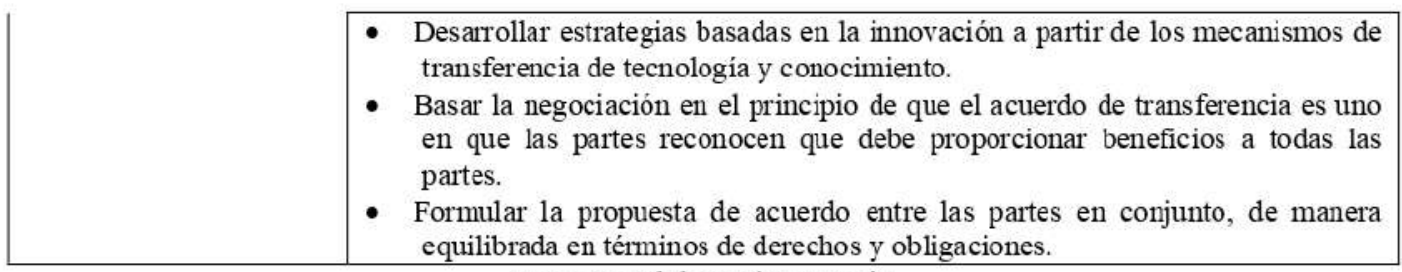

Fuente: Elaboración propia

Objetivo Estratégico No. 2: Incrementar las capacidades tangibles e intangibles del negocio exportador mediante una efectiva adaptación tecnológica (ver cuadro 5).

Cuadro 5. Acciones de la estrategia de adaptación

\begin{tabular}{|c|c|}
\hline PROCESO CLAVE & ACCIONES ESTRATÉGICAS \\
\hline $\begin{array}{l}\text { Adaptación de } \\
\text { tecnología }\end{array}$ & $\begin{array}{l}\text { - Planificar los procesos de adaptación tecnológica de acuerdo con las } \\
\text { condiciones internas del negocio y las características del entorno. } \\
\text { - Realizar estudios de mercado para determinar su tamaño, como componente } \\
\text { económico fundamental del proceso. } \\
\text { - Incrementar la disposición gerencial como catalizador del proceso de } \\
\text { adaptación de la tecnologia. } \\
\text { - Capacitar al talento humano en el rediseño y adaptación de planta, equipo, } \\
\text { procesos y mejora de productos. } \\
\text { - Documentar las experiencias anteriores de procesos de adaptación tecnológica. } \\
\text { - Potenciar alianzas con centros de I+D y universidades para el intercambio de } \\
\text { conocimientos en estrategias de ingeniería inversa para la adaptación } \\
\text { tecnológica. } \\
\text { - Participar en proyectos de investigación conjunta, patrocinada por la empresa, } \\
\text { lo cual le permite el acceso exclusivo al conocimiento y los resultados, con el } \\
\text { objetivo de desarrollar innovaciones incrementales o resolver problemas } \\
\text { concretos del negocio exportador. } \\
\text { Establecer vínculos con el sector académico, que permita la transferencia del } \\
\text { conocimiento tácito de investigadores y universitarios hacia el sector } \\
\text { empresarial. }\end{array}$ \\
\hline
\end{tabular}

Fuente: Elaboración propia

Objetivo Estratégico No. 3: Fomentar la apropiación social del conocimiento tecnológico dada la asimilación del mismo como elemento de inteligencia competitiva (ver cuadro 6).

Cuadro 6. Acciones de la estrategia de asimilación

\begin{tabular}{|l|l|}
\hline PROCESO CLAVE & \multicolumn{1}{c|}{ ACCIONES ESTRATÉGICAS } \\
\hline & $\begin{array}{c}\text { Promover el aprovechamiento del conocimiento en el que el negocio } \\
\text { profundiza una vez transferida la tecnología. }\end{array}$ \\
& $\begin{array}{l}\text { Asumir la asimilación del conocimiento como el medio para incrementar la } \\
\text { curva de aprendizaje organizacional y la inteligencia competitiva. }\end{array}$ \\
Gestionar la información necesaria para comprender la tecnología adquirida \\
(documentación técnica, manuales de uso,...)
\end{tabular}




\section{Transferencia de tecnología y conocimiento en el sector exportador de bovinos}

\begin{tabular}{|l|l|}
\hline Diagnosticar necesidades de formación relacionadas con el conocimiento \\
requerido de la tecnología transferida. \\
Elaborar planes de formación y actualización al personal que permitan estar al \\
dia en cuanto a avances del conocimiento y la tecnología inicialmente \\
adquirida. \\
Establecer un presupuesto destinado a la formación permanente del talento \\
humano. \\
Diseñar un plan de revisión frecuente de los paquetes tecnológicos para \\
determinar el estado de la información y promover las actualizaciones \\
necesarias,
\end{tabular}

Fuente: Elaboración propia

\section{Conclusiones}

Se concluye que, la propuesta formulada a través de acciones estratégicas orientadas a la negociación y adaptación tecnológica, como a la asimilación del conocimiento, se convierte en una guía a las empresas del sector exportador de ganado bovino para alcanzar niveles más altos en la curva de aprendizaje organizacional con mayor empoderamiento, consecuencia de la apropiación social del conocimiento tecnológico transferido y capitalizado como parte de su sistema de inteligencia competitiva.

Se recomienda emprender acciones gerenciales que fomenten la consolidación de una cultura hacia la gestión tecnológica, que propenda el desarrollo de habilidades de planificación, redes de productividad, capacidades de innovación, apropiación del conocimiento tecnológico e inteligencia competitiva.

\section{Referencias}

Avendaño, E. (2017). El uso de la transferencia de tecnología en el sector empresarial: de la innovación a la apropiación del saber. (Trabajo de maestría). Pontificia Universidad Javeriana, Santiago de Cali, Colombia.

Beraza J. \& Rodríguez, A. (2010). Estructuras de intermediación para la transferencia de conocimiento universitario: las oficinas de transferencia de tecnología. Propiedad intelectual, 152-176.

Borbón, C. \& Arvizu, M. (2015). Contraste empírico de la transferencia de tecnología en tres empresas de agricultura protegida en México. Nova Scientia 7(3), 464-388.

Correa, Z. (2012). Modelo de caracterización de estrategias de innovación tecnológica en empresas universitarias de base tecnológica: un estudio de caso. (Trabajo de maestría). Universidad Tecnológica de Bolivar, Cartagena de Indias, Colombia.

Díaz, J. A., Borroto, O., Suárez, J., \& Castillo, E. (2005). La transferencia de tecnologías en el sector ganadero latinoamericano. La experiencia cubana. Revista Cubana de Ciencia Agrícola, 39, 407-414.

Espinal, C. F., Martínez Covaleda, H. \& Amézquita, J. E. (2006). La cadena de ovinos y caprinos en Colombia. Bogotá: Ministerio de Agricultura y Desarrollo Rural.

Finston, S. (2011). Manual de buenas prácticas, transferencia de tecnología hacia los países de ingresos medios. Disponible en: http://pipra.fia.cl/media/9326/f2 1 finston vf 13-04-2011.pdf

Galindo, J., Sanz, P., \& De Benito, J. J. (2011). La universidad ante el reto de la transferencia del conocimiento 2.0: Análisis de las herramientas digitales a disposición del gestor de transferencia. Investigaciones Europeas de Direccion Y Economia de La Empresa, 17(3), 111-126. https://doi.org/10.1016/S1135-2523(12)60123-3

Galindo Gutiérrez, J. L. (2014). Plan exportador en los sistemas semi-estabulado de ganado de carne bovino en productores de la vereda el guayabo-Pto. Salgar, Cundinamarca (Tesis Doctoral), Universidad del Rosario, Bogotá.

IPSA SCIENTIA - Vol. 4 No 1 - 2019 


\section{Transferencia de tecnología y conocimiento en el sector exportador de bovinos}

Geizzelez, M. \& Linares, J. (2016). Transferencia tecnológica para la vinculación con las comunidades en el Programa Nacional de Formacion en Informatica. REVECITEC, 6(2), 1-14.

González-Sabater, J. (2011). Manual de transferencia de tecnología y conocimiento. Alicante, España: Instituto de transferencia de tecnología y conocimiento.

González, K. (2015). Transferencia tecnológica en las universidades públicas del Cesar, Desarrollo Gerencial, Revista de la Facultad de Ciencias Económicas, Administrativas y Contables de la Universidad Simón Bolivar 7(1), 85-101.

Guerrero, M. \& Urbano, D. (2012). Transferencia de conocimiento y tecnología: Mejores prácticas en las universidades emprendedoras españolas. Gestión y política pública, 21 (1): 109-110.

Hernández, R. Fernández, C. \& Baptista, P. (2014). Metodología de la investigación. 3ra edición. Chile: Editorial McGraw-Hill Interamericana.

Herrera, J. \& Giraldo, O. (2010). Knowledge appropriation between private education institutions: CUPI2 project transfer from uniandes to uniminuto. JISTEM Journal of Information Systems and Technology Management, 7(1), 33-70. https://doi.org/10.4301/S1807-17752010000100002

Hidalgo, A.; León, G. \& Pavón, J. (2002). La gestión de la innovación y la tecnologia en las organizaciones. Madrid, España: Ed. Pirámide.

Hurtado, J. (2015). El proyecto de investigación, Comprensión holistica de la metodología y la investigación. Caracas, Venezuela: Sypal Quiron ediciones.

León, J., López, S., \& Sandoval, S. (2009). Actividades de transferencia del conocimiento de los investigadores académicos en el estado. Revista de La Educación Superior, XXXVIII(151), 85-111.

Linares-Morales, J. \& Geizzelez-Luzardo, M. (2016). Políticas científicas y tecnológicas para la gestión del conocimiento en instituciones universitarias. IPSA SCIENTIA: Revista Cientifica Multidisciplinaria, 1(1), 10-17. Recuperado a partir de https:/latinjournal.org/index.php/ipsa/article/view/889

Lombana Coy, J., Martínez Martelo, D. N., Rubio Oquendo, J. A., Castrillón Cifuentes, J., \& Marino Manga, W. (2013). Caracterización del sector ganadero del Caribe colombiano. Barranquilla: Editorial Universidad del Norte.

López, E. (2011). Metodología de la investigación: Guía instruccional. Caracas: Universidad NacionalAbierta.

López M., Mejía J. \& Schmal R,. (2006). Un acercamiento al concepto de transferencia de tecnología en las universidades y sus diferentes manifestaciones. Panorama Socioeconómico, 70-81.

Muñoz, C. (2018). Estrategias innovadoras para la generación de nuevos proyectos tecnológicos y educativos. Caso de estudio: Clear Minds Consultores Cía. Ltda. (Trabajo de maestría). Universidad Andina Simón Bolivar, Quito, Ecuador.

Oviedo-Socarrás, T., Vargas-Viloria, M., \& Cardona, J. A. (2016). Frecuencia de lesiones macroscópicas en pulmones de bovinos tipo exportación faenados en el frigorífico del municipio de Montería, Colombia. Revista de la Facultad de Medicina Veterinaria y de Zootecnia, 63(1), 30-38.

Reyes Gutiérrez, P. M., Anaya Rincón, C. A., \& Rojas Ávila, C. J. (2018). Operaciones para mejorar la competitividad internacional del sector cárnico bovino colombiano, estudio de caso Estados Unidos. (Trabajo de Grado). Universidad Agustiniana, Bogotá.

Reyes, J. (2010). Transferencia tecnológica en institutos universitarios de tecnología. Revista Electrónica Facultad de Ingeniería UVM, 4(2): 280-296.

IPSA SCIENTIA - Vol. 4 N $1-2019$ 
Robledo, J. (2013). Introducción a la Gestión de la Tecnología y la Innovación. Medellín, Colombia: Universidad Nacional de Colombia.

Rubiralta, M. (2003). Transferencia a las empresas de la investigación universitaria: documento de trabajo. Madrid, España: Academia Europea de Ciencias y Artes.

Solano, E, Arzola, M; Durán, M. \& Chacón, F. (2013). Modelo para transferencia de tecnología en empresas públicas. Caso de estudio: Siderúrgica Alfredo Maneiro SIDOR. Ingeniería Industrial. Actualidad y Nuevas Tendencias, III (10), 23-38.

Vargas, L. (2017). Plan de transferencia tecnológica para sistema de comunicaciones unificadas en el sector textil de Bucaramanga. Caso de estudio: Comertex S.A. (Trabajo de maestría). Universidad Nacional de Colombia, Medellín.

Vázquez, E. (2017). Transferencia del conocimiento y tecnología en universidades. Iztapalapa Revista de Ciencias Sociales y Humanidades, 83(38), 75-95.

Villarreal Castro, M. (2015). Análisis de los temas de salubridad y logística que Colombia debe abordar para exportar carne de bovino a la República Popular China (Tesis Doctoral), Universidad del Rosario, Bogotá.

Wehn, U., \& Montalvo, C. (2016). Knowledge transfer dynamics and innovation: Behaviour, interactions and aggregated outcomes. Journal of Cleaner Production, 1-13. https://doi.org/10.1016/j.jclepro.2016.09.198 